\title{
Report
}

\section{Improving Timeliness in the Bronchoscopy Workflow: From Admission to Discharge, A Single-Center Quality Initiative}

\author{
Kevin Charles*, Darien Davda, Kevin Langlois, Naser Mahmoud, Ashley Ferraro, Robert Holladay, \\ Satish Kalanjeri, Robert Walter
}

Louisiana State University Health Sciences Center, Shreveport, Louisiana, United States

\section{Email address:}

kc.kevincharles@gmail.com (K. Charles)

${ }^{*}$ Corresponding author

\section{To cite this article:}

Kevin Charles, Darien Davda, Kevin Langlois, Naser Mahmoud, Ashley Ferraro, Robert Holladay, Satish Kalanjeri, Robert Walter. Improving Timeliness in the Bronchoscopy Workflow: From Admission to Discharge, A Single-Center Quality Initiative. Science Journal of Clinical Medicine. Vol. 8, No. 4, 2019, pp. 44-48. doi: 10.11648/j.sjcm.20190804.13

Received: May 22, 2019; Accepted: August 10, 2019; Published: August 23, 2019

\begin{abstract}
Several delays arise when bronchoscopy and endoscopy procedures are scheduled in the same room. Turnover time, workflow, and technician and/or anaesthesia availability all play a role in the length of stay of a patient for this same-day procedure. A planned procedure is elective and should be lucrative for a patient, their families, and staff. This quality improvement initiative aimed at improving bronchoscopy workflow with hopes of significantly decreasing admission to discharge time for the patients. Satisfaction surveys and post-discharge follow-ups were conducted parallel to this quality initiative. Analysis of the data identified potential areas for future endeavors aimed at decreasing hospital costs with improved patient satisfaction and goals of improving the fellows experience at a teaching institution.
\end{abstract}

Keywords: Bronchoscopy, Length of Stay, Efficiency, Delays, Patient Satisfaction, Fellowship

\section{Introduction}

Judicious bronchoscopy workflow in an educational teaching facility simultaneously provides a positive patient experience and rotation experience for the fellow. However, prior studies have shown such facilities can have various degrees of complications and setbacks; such complications can be divided into intrinsic complications (by being a teaching facility for novice pulmonary fellows, certain complications arise from procedures) [1], and extrinsic complications (delays and setbacks not intrinsically related to training). A thorough literature search presents many identifiable sources for extrinsic complications; staff availability, coordination of patient transportation, and accessibility to equipment and medications have been cited as common reasons for inefficiency in a procedural suite [2]. Over the last few years at our institution there have been several obstacles that have stunted our bronchoscopy throughput. Consequentially patients have delayed diagnoses, and a backlog of pended procedures usually always persists each month. Our literature search also recognized various quality improvement studies aimed at improving efficiency in the bronchoscopy procedure suites. Various projects have utilized similar measures from surgical specialties to determine if similar yields could be obtained, such as a surgical checklist prior to each procedure [3]. For our institution, we designed a quality improvement project with a goal of improving patient turnover and decreasing transit time between scheduled bronchoscopy cases. By decreasing transit time, we hoped to increase the volume of procedures performed each month, thereby improving the fellows experience during the bronchoscopy procedure rotation, as determined in prior reviews [4]. Additionally, we hoped to improve patient satisfaction during their "same-day surgery" admission by decreasing their waiting time and their length of stay. We set a concrete goal of completing 2 cases/day, prior to noon.

\section{Rationale}

We describe the design of a system intervention focused on improving the workflow in the bronchoscopy suite. By 
striving to understand the barriers affecting activity system components, we were able to develop a system/workflow that addressed decreasing patient length of stay, improving fellows' rotation experience, and decreasing work hours for ancillary staff. Interventions thereby succeeded at improving patient satisfaction, educational environment, and hospital cost, similarly to prior studies reducing time between operations in a surgical suite [5].

\section{Materials and Methods}

Nurse supervision, fellows, attendings and procedure technicians compiled data. The following data were obtained for assessment:

1. Transition Times: defined by the following segments-(admission to rooming patient time), (room to procedure start), (room to discharge) and (admission to discharge).

2. These data were then stratified into transit time before and after our Pulmonology department had established our own bronchoscopy suite. Prior to our suite, we were sharing an intervention room with the Gastroenterology department.

Our quality improvement data analysis identified delays between when patient is in room and when case actually starts. An excel spreadsheet was used to compile data and identify delays. Wilcoxon Rank Sum tests were performed to statistically analyze the data for significant differences, if present $[6,7]$.

\section{Results}

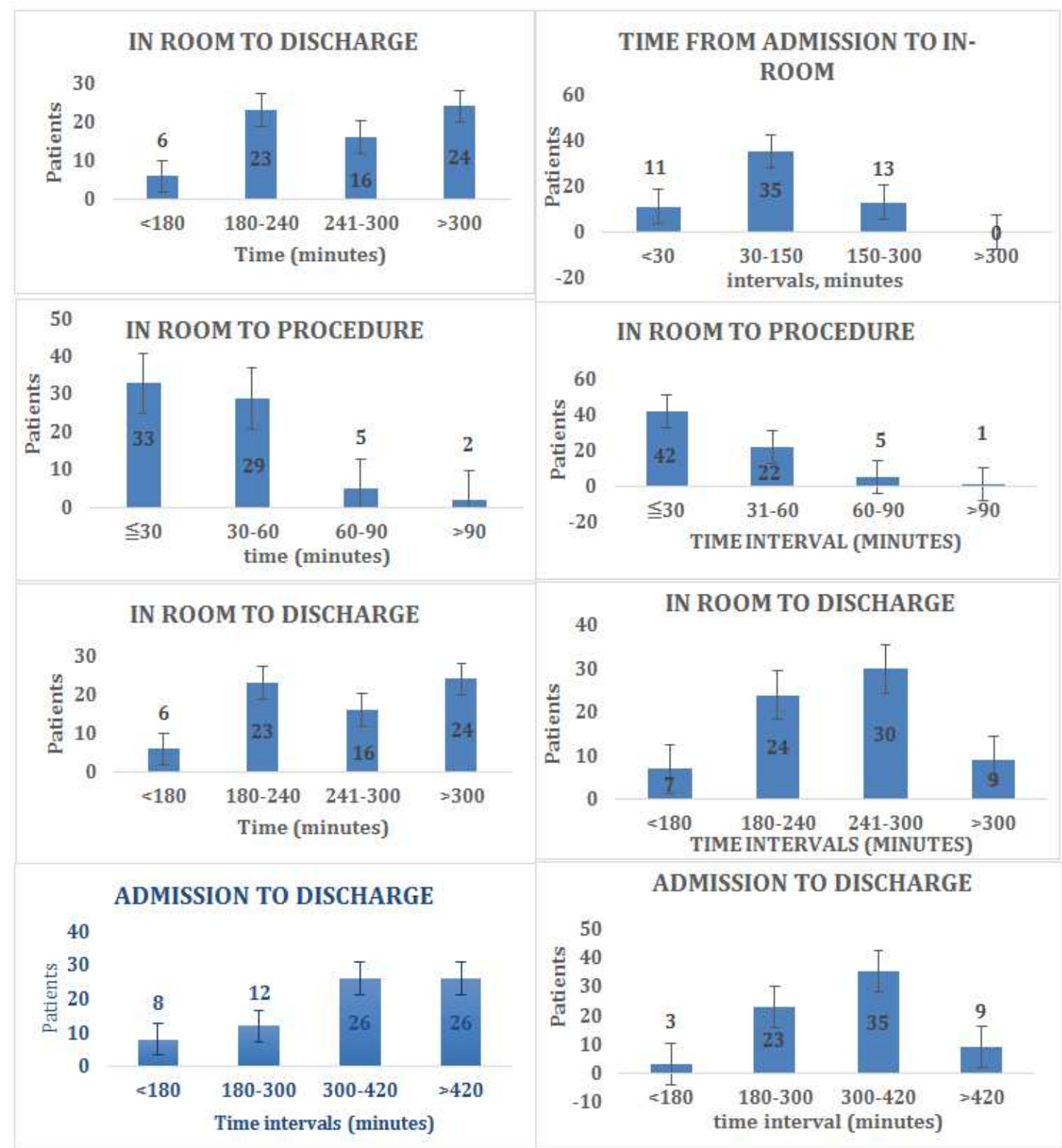

Figure 1. (Left column) Results of bronchoscopy workflow from admission to discharge with SHARED ROOM (right column) Results of bronchoscopy workflow from admission to discharge with DEDICATED ROOM. 
Our first "procedure start" time improved by 1.3 hours when comparing before and after the dedicated room was established. "Admission to in room" time was a time point that provided interesting analytical data, as did the "Admission to Discharge" time. "In room to procedure start" time was consistent in both groups. No intervention was implemented to decrease this transit time. Endoscopy staff and fellows participated in a survey that asked them to determine what would be an expected transit time from when a patient enters the room to procedure start time. Answers were compiled to give a rounded average of $\sim 20$ minutes. "In room to discharge" time was averaged at $362 \mathrm{~min}$ in the shared room, with 24 patients having a length of stay $>300$ min after entering the room. "In room to discharge" time was averaged at $248 \mathrm{~min}$, with 9 patients having a length of stay $>300 \mathrm{~min}$.

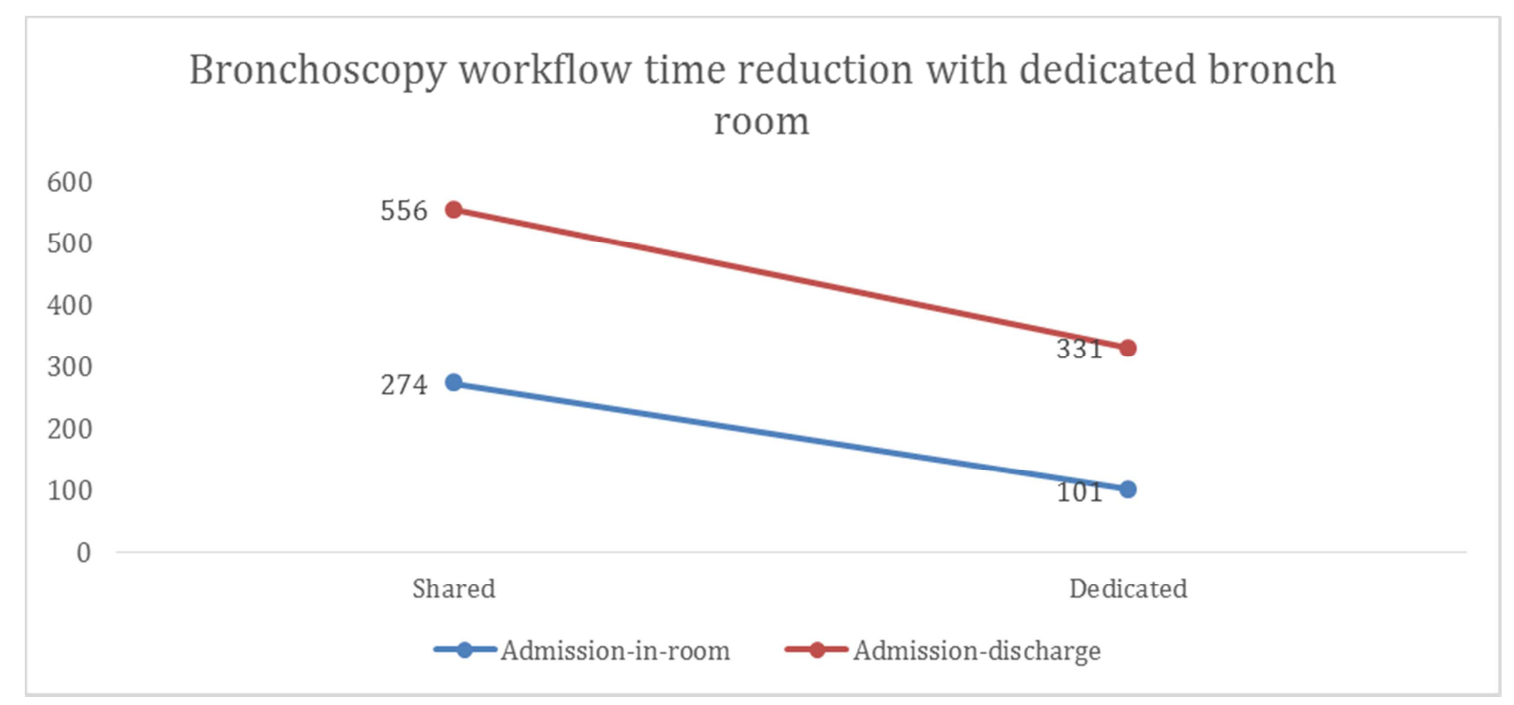

Figure 2. Reduction in bronchoscopy workflow time with implementation of a dedicated bronchoscopy suite. Both categories (admission to rooming time for inpatient), (admission to discharge), produced the most notable declines in utilized time.

\section{Statistical Analysis}

Table 1. Statistical Assessment of Bronchoscopy Categories and Data Collection.

\begin{tabular}{ll}
\hline Statistical Assessment of Bronchoscopy Study using Wilcoxon Rank Sum Test \\
\hline Admission to In-Room, Retrospective and Prospective & Test Statistic R1 $=5018.5, \mathrm{Ho}=0, \mathrm{Ha} \neq 0 . \mathrm{z}$ statistic $=3.222, \mathrm{p}$-value: 0.0013 \\
In-Room to Procedure, Retrospective and Prospective & Test Statistic R1 $=4866, \mathrm{Ho}=0, \mathrm{Ha} \neq 0 . \mathrm{z}$ statistic $=0.152, \mathrm{p}$-value: 0.8795 \\
In-Room to Discharge, Retrospective and Prospective & Test Statistic R1 $=5175, \mathrm{Ho}=0, \mathrm{Ha} \neq 0 . \mathrm{z}$ statistic $=1.453$. p-value: 0.1461 \\
Admission to Discharge, Retrospective and Prospective & Test Statistic R1 $=5607, \mathrm{Ho}=0, \mathrm{Ha} \neq 0 . \mathrm{z}$ statistic $=1.873$. p-value: 0.0611 \\
\hline
\end{tabular}

As seen from the assessment, there was enough evidence to contribute to a statistically significant difference in time between the retrospective and prospective data (before and after the bronchoscopy suite was established) for admission to in-room timing, but not for in-room to procedure, in-room to discharge, or admission to discharge.

\section{Discussion}

As part of our quality improvement study, we became familiar with prior investigations in efficiency assessments and quality measures pertaining to bronchoscopy. For instance, one prior study reviewed patient satisfaction with their experiences during flexible bronchoscopy. This study emphasized any future endeavors clinicians implement that could potentially improve bronchoscopy technique or efficiency would consequentially increase patient satisfaction and follow-up if indicated [8]. Additionally as touched upon previously, prior studies have incorporated protocol from other specialties; Thillai et al devised a bronchoscopy procedure checklist following several PDSA cycles in order to improve efficiency, which was determined from clinician satisfaction. A key takeaway from this study is the incorporation of such guidelines or protocol, but with a goal of reducing waiting times and improving satisfaction among patients and proceduralists within the same day of the operation. By establishing our own bronchoscopy suite, we were able to initiate development of protocols for our own operation schedules and systems management [3]. Prior to establishing our own bronchoscopy suite, some of the conflicts our program experienced included ongoing conflict with gastroenterology service regarding scheduling different operations in one room, identifying whether our cases are being posted in a timely manner, and endoscopy technicians not experienced in bronchoscopies affecting workflow in the room due to inconsistencies in completing appropriate preoperative tasks. The advent of a "dedicated bronchoscopy room" allowed for significantly more flexibility within the 
department. Dedicated bronchoscopy technicians made for efficient workflow during the procedure. On average our procedure time decreased by 13 minutes across procedures such as diagnostic EBUS and navigational bronchoscopy. We did not include tumor debulking, rigid bronchoscopies, or bronchial thermoplasty in either group. Most importantly, we believe our intervention of creating a dedicated bronchoscopy suite improved patient satisfaction by decreasing time to discharge for each patient, decreasing waiting time before procedure start and also improved the fellows experience by increasing the number of procedures completed in 24 hours. Krvavac et al determined the types of delays their institution procured within their bronchoscopy suite, with particular attention to procedure start time delays, and potential areas for interventions to reduce inefficiency. For intervention, they increased awareness and education among personnel and designing the process to reduce time delays, which led to statistically significant improvement [9]. Similarly, we are devising protocols for our faculty to utilize for procedural days in hopes of reducing delays and inefficiency. One other important facet of reducing the time in transition from bed to procedure, will be the goal of focusing future improvement on reduction of time during procedure that otherwise would expose patients to significant levels fluoroscopic radiation. Pasnick et al demonstrated in a quality improvement project that increased awareness of fluoroscopy radiation and produced a reduction in fluoroscopy time that would have otherwise been dangerous for the patient [10]. As mentioned previously, while training facilities can provide excellent clinical education, increased procedural time can lead to delays and exposure for complications. By improving transition times between operations, training clinicians can focus their quality improvement (both directly as a project and indirectly in terms of improving their prowess) on time within the procedure, especially fluoroscopy time. Moving forward we hope to continue optimizing the efficiency of workflow in the bronchoscopy suite. We hope to implement written instructions for patients when seen in clinic providing pre-procedure instructions, procedure time, location, and date while further decreasing delays. The current goal to decrease patient length of stay was obtained and is most notably illustrated by the "admission to discharge" time. This transit time decreased from 9 hours 14 minutes, to 5 hours 31 minutes for a difference of 3 hours 43 minutes. Patient length of stay was decreased by almost 4 hours making their "same-day surgery" day considerably shorter and less tedious due to decreased waiting times. Based off our analysis, certain factors seen in the literature review such as staffing deficiency, contributed to delays in procedure efficiency [11].

The fellows experience was improved when some sort of briefing for each case was done prior to the procedure start so that all parties involved were on the same page regarding procedure objectives and manner in which objectives would be achieved. A survey of first and second year fellows revealed that pre-procedure briefing was an important part of the fellows' educational experience. Moving forward, we hope to devise educational sessions for fellows and bronchoscopy technicians so that all team members fully understand the machinery and instrumentations used for the different pulmonary procedures performed in the bronchoscopy suite. We also hope to create an orientation packet for incoming fellows so that expectations during the bronchoscopy rotation are made clear. It would be beneficial for future quality improvement measures regarding bronchoscopy efficiency follow parameters similarly to the multi-institutional "AquIRE" program, in order to evaluate clinical outcomes and complication rates between different procedures, such as pneumothorax rates with transbronchial biopsies [12]. Additionally, future projects will include decreasing this transit time by having thorough communication with anaesthesia to better coordinate this transition. Incorporating additional staff such as anesthesia would be beneficial for this study, as future measures could focus on patient satisfaction regarding pain control within the procedure, as a similar quality improvement project determined tolerance for pain and appropriate airway anesthesia contributed to patient reluctance to undergo bronchoscopic re-examination if indicated [13]. Importantly, similar utilization of a questionnaire for patient and clinician satisfaction could be ideal for milestone objectives. Lastly, interventional pulmonology is a vastly expanding and changing field $[14,15]$. As advancement in technology continues and novel techniques or equipment such as optical coherence tomography or confocal microscope become more widely used, safety profiles and complication rates may alter, and implementing basic protocols now could serve as a cornerstone for future work.

\section{Conclusions}

In conclusion we have identified delays in the bronchoscopy suite workflow and intervened to successfully develop more efficiency in this area. By decreasing transit times and improving workflow in the bronchoscopy suite, the case throughput has increased tremendously. We have met our main goals to decrease patient length of stay during their "same-day surgery" stay and improve the fellows' educational experience during the bronchoscopy rotation block.

\section{References}

[1] Strather DR, MacEachern P, Chee A, et al. Trainee Impact on Procedural Complications: An Analysis of 967 Consecutive Flexible Bronchoscopy Procedures in an Interventional Pulmonology Practice. Respiration 2013; 85: 422-428.

[2] Strack L, Brawner E, Bruns A, Devulapally K, et al. Delays and efficiency in the bronchoscopy suite perception meets reality. J Bronchol Intervent Pulmonol. 2010. Volume 17, Number 3.

[3] Thillai M, Powrie D. Quality improvement in respiratory medicine: designing and implementing a bronchoscopy checklist at Southend University Hospital. BMJ Quality Improvement Reports. 2013. u201878.w997 doi: 10.1136/bmjquality.u201878.w997. 
[4] Fielding, David I., et al. "Achieving Competency in Bronchoscopy: Challenges and Opportunities - Fielding - 2014 - Respirology - Wiley Online Library.” Respirology, John Wiley \& Sons, Ltd (10.1111), 1 Apr. 2014, onlinelibrary.wiley.com/doi/10.1111/resp.12279.

[5] Harders $M$, Malangoni MA, Weight $S$, et al. Improving operating room efficiency through process redesign. Surgery. 2006; 140: 509-514.

[6] Arts, D De Keizer NF, Bosman R, De Jonge E, Joore J. Training in data definition improves quality of intensive care data. Critical Care. 7: 129-184. 2002.

[7] Arts D, De Keizer NF, Scheffer GT. Defining and improving data quality in medical registries: a literature review, case study, and generic framework. J Am Med Inform Assoc 9, 6: P 600-11. 2002.

[8] Lechtzin N, Rubin HR, White P et al. Patient Satisfaction with Bronchoscopy. American Journal of Respiratory and Critical Care Medicine. 2002; 166 (10).

[9] Krvavac A, K Ghassan, P Patel et al. Evaluating and Improving Efficiency of Patient Flow in the Bronchoscopy Suite in a Tertiary Care Center: A QI Project. Quality Management in Health Care. 27 (1): 33-38, January 2018.
[10] Pasnick S, Hayes M, Salvaggio $M$ et al. A Quality Improvement Project Aimed at Reducing Fluoroscopy Time During Bronchoscopy Significantly Decreases Total Radiation Exposure.

[11] Yong E, Zenkova O, Saibil F, et al. Efficiency of an endoscopy suite in a teaching hospital: delays, prolonged procedures, and hospital waiting times. Gastrointest Endosc. 2006; 64: 760-764.

[12] Cox K, TJ Harkin. Quality Improvement Initiative in Bronchoscopy Inspired by the Acquire Program. Am J Respir Crit Care Med 2017; 195: A1657.

[13] Fujimoto K, Ishiwata T, Kasai H, Terada J, Shionoya Y, Ikari J, et al. (2018) Identification of factors during bronchoscopy that affect patient reluctance to undergo repeat examination: Questionnaire analysis after initial bronchoscopy. PLoS ONE 13 (12): e0208495.

[14] Leong, Steven et al. "Diagnostic bronchoscopy--current and future perspectives." Journal of thoracic disease vol. 5 Suppl 5, $\begin{array}{llll}\text { Suppl } & 5 & \text { (2013): } & \text { S498-510. }\end{array}$ 10.3978/j.issn.2072-1439.2013.09.08.

[15] Ernst, A et al. A Multicenter Prospective Advanced Diagnostic Bronchoscopy Outcomes Registry. CHEST. 138 (1); 165-170. 